\title{
Inter- and Intra-Relationships between Communication Coordination and Cooperation in the Scope of the 3C Collaboration Model
}

\author{
Hugo Fuks ${ }^{1}$, Alberto Raposo ${ }^{1}$, Marco Aurélio Gerosa ${ }^{2}$, \\ Mariano Pimentel $^{3}$, Denise Filippo ${ }^{1}$, Carlos Lucena ${ }^{1}$ \\ ${ }^{1}$ Computer Science Department, Pontifical Catholic University of Rio de Janeiro, Brazil \\ \{hugo,denise,lucena\}@inf.puc-rio.br,abraposo@tecgraf.puc-rio.br \\ ${ }^{2}$ Computer Science Coordination, Vila Velha University,Brazil \\ gerosa@inf.puc-rio.br \\ ${ }^{3}$ Department of Applied Informatics, Federal University of the State of Rio de Janeiro, Brazil \\ pimentel@uniriotec.br
}

\begin{abstract}
Based on the $3 C$ Collaboration Model, this article descries the mapping of a variety of collaboration forms onto inter-relationships between communication, coordination and cooperation. In order to investigate how to provide computational support for these three functional collaboration dimensions the analysis shifts from the inter-relationships between these three dimensions to their intra-relationships. Finally, Gestalt psychology principles are used to discuss the suitability of the approach to human perception.
\end{abstract}

Keywords: 3C Collaboration Model, Communication, Coordination, Cooperation.

\section{Introduction}

Along more than two decades of existence as an area of study, groupware has been classified, analyzed and developed based on diverse criteria, such as time/space taxonomy, application domain, underlying technology, group tasks, modes of interaction and functionality. The objective of this paper is to focus on one of these criteria, namely functionality, in order to outline a conceptual framework for classifying, analyzing and developing collaborative systems.

In this article, collaboration is functionally divided into 3 dimensions: communication, coordination and cooperation. Communication or conversation consists in the exchange of messages and in negotiations among people; coordination consists in the management of people, their activities and their resources; and cooperation is the production that takes place in the shared workspace. This model of collaboration was originally proposed by Ellis et al. [5], with some differences in terminology: what Ellis calls "collaboration" here is characterized as cooperation.

In the continuation of this article it is investigated the literature related to the $3 \mathrm{C}$ model. Then, a deeper understanding of how the model can support collaboration analysis and development is provided based on the inter- and intra-relationships between these 3 dimensions. Finally, based on the configuration of the user interface and on Gestalt Psychology studies of form visualization, it is indicated how individuals that use systems based on this model alternate among acts of communication, coordination and cooperation.

\section{The 3C model}

Among the groupware functional models, the 3C model and its variations are probably the most widespread used. The most common use of the model is to classify collaborative applications. As an example (Figure 1), Borghoff \& Schlichter [2] present a triangular taxonomy where applications are positioned according to their support to each $\mathrm{C}$.

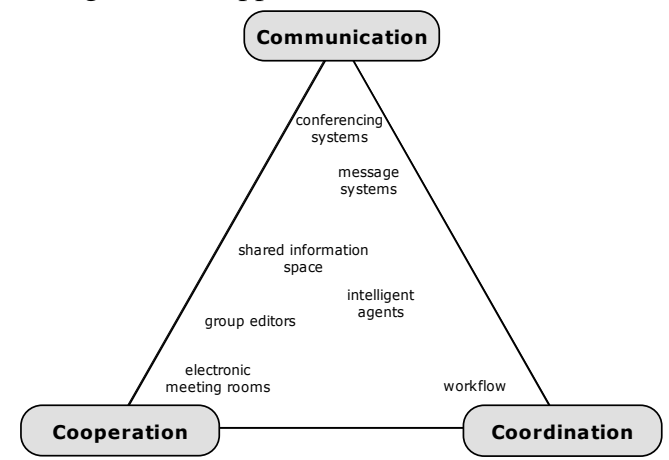

Figure 1. Classification of applications based on the $3 \mathrm{C}$ model

Still regarding the use of the 3C model for classification purposes, Magnusson \& Svensson [13] design the computational support for groups of students using the $3 \mathrm{Cs}$ as the basis for classifying tools.

The 3C model is also used as an analysis tool for different purposes. Bretain et al. [3] use the Cs to 
analyze and interview professional groups whose activities are realized out of the office in order to define an adequate multimedia computational support for each group. A collaborative system evaluation approach based on a model similar to the $3 \mathrm{C}$ is presented in [16].

There are also a few attempts to use the $3 \mathrm{C}$ model as a basis for the implementation of collaborative systems. An example is the model used by Clover, which defines 3 classes of functionalities named communication, coordination and production [11]. It is similar to the $3 \mathrm{C}$ model in terms of functional specification of collaborative systems, insofar as both deal with the 3 classes of functionalities that must have computational support in groupware.

Also regarding implementation, Bandinelli et al. [1] use the three dimensions of the $3 \mathrm{C}$ model to improve computational support for software processes, mainly in communication and coordination aspects. According to these authors, these aspects are not adequately treated by traditional processes, which are mainly focused on the coordination of groups of developers.

There are also a couple of other functional models for collaborative applications that may be considered as variations of the $3 \mathrm{C}$ model for specific purposes. For example, Malone \& Crowston [14] consider a model with 3 dimensions: coordination, communication, and group decision making. According to our understanding, Malone's model is actually a deeper analysis of the coordination dimension of collaboration. Weiseth et al. [19] consider the collaboration process as composed of three sub-processes, namely, coordination, production, and decision-making. According to them, production is related to the creation and sharing of information and knowledge, as well as to the distribution of information through communication channels. Therefore, their definition of production comprises the dimensions of communication and cooperation of the $3 \mathrm{C}$ model.

Given that most of current collaborative systems encompass the $3 \mathrm{Cs}$, a static visualization informs the main purpose of the system without revealing the other functionalities supported by them. In order to provide an integrated analysis, in the next section the interrelationships between the $3 \mathrm{Cs}$ are uncovered.

\section{The 3Cs mapped onto collaborative systems: Inter-relationships}

In this section, the $3 \mathrm{C}$ triangle is enlivened by examples of representative collaboration forms found in the literature. These examples illustrate 6 possible cycles starting from the $\mathrm{C}$ that indicates the main purpose of the system. Each $\mathrm{C}$ in the cycle represents a functional phase in the use of the system and the arrows represent the nature of the inter-relationship.

The first example of the $3 \mathrm{C}$ model focuses on the group work domain as represented in Figure 2, where conversation aims at action; while they communicate, people negotiate and take decisions, and while they coordinate, people deal with conflicts and organize their activities in a way that prevents the waste of efforts. Cooperation is the joint operation of the members of the group in a shared space who carry on tasks when generating and manipulating cooperation objects. The need to renegotiate and take decisions regarding unexpected situations that occur during cooperation demands a new round of communication that, in turn, will demand coordination to reorganize the tasks to be executed during cooperation.

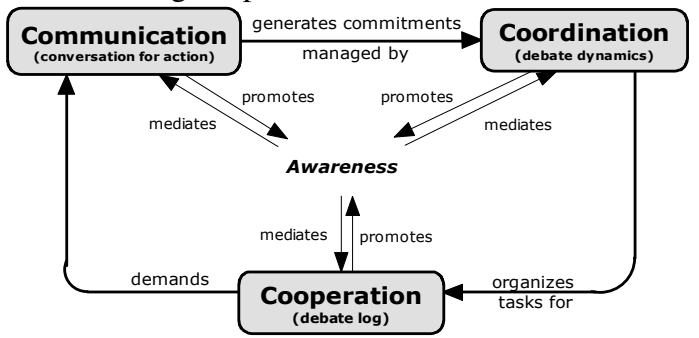

Figure 2. 3Cs in Conversation for Action

Figure 3 illustrates an adaptive workflow [18]. When executing daily tasks pre-articulated by a workflow, members of the group feel the need to modify what was previously agreed upon. A counter-example is the classical industrial assembly line, where employees are not expected to negotiate the execution of the upcoming tasks based on the results obtained in the execution of the previous ones.

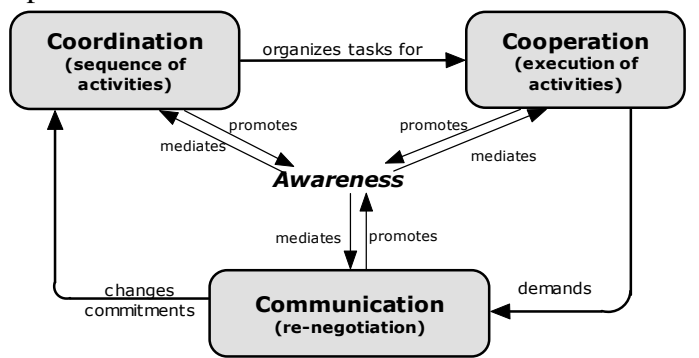

Figure 3. 3Cs in Adaptive Workflow

Social websites such as, for example, Orkut (http://www.orkut.com), have their cycle exemplified in Figure 4. Members of a community place their profiles in a common area, which raise the attention and the interest of some of these members, who exchange messages, organize parties, date, etc., and, eventually, invite new members to join the community.

For Media Spaces [12], multimedia spaces whose objective is the informal communication among people, the $3 \mathrm{C}$ model is exemplified in Figure 5. Media Spaces is the space literally shared through a distributed video such as, for example, the RAVE system developed at EuroPARC [9]. As it is directed to informal communication, its main objective is to create opportunities for casual meetings which are coordinated by the established social protocol, for example, accessing information about the availability of remote 
peers. These meetings propitiate conversations that take place through the use of the means provided by the system itself, or available in other forms.

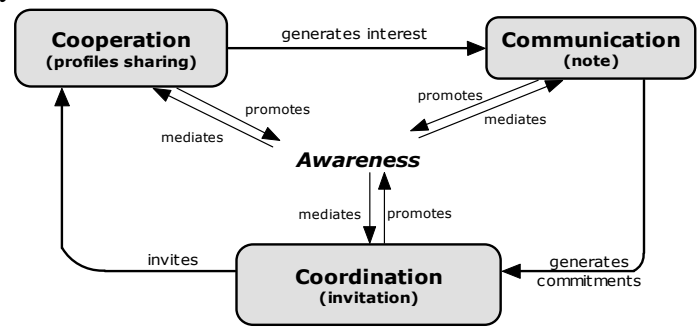

Figure 4. 3Cs in Networking Sites

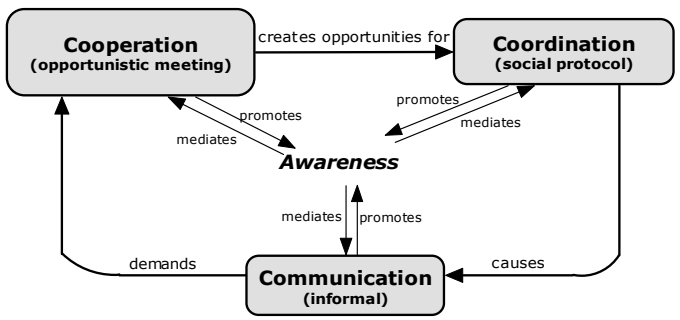

Figure 5. 3Cs in Media Spaces

The main reason to use a family calendar is the scheduling of family activities (Figure 6). Members of a modern family have a variety of conflicting interests that can render arrangements agreed upon during the evening useless the following morning. In order to restore family coordination, negotiation among its members is necessary. "This process involves verifying what has already been scheduled, (...) and the negotiation of incumbencies, schedules and other responsibilities is necessary" [4]. The reconciliation achieved after the negotiation round is registered in the shared calendar. But as life never stops, in the following morning the cycle starts again.

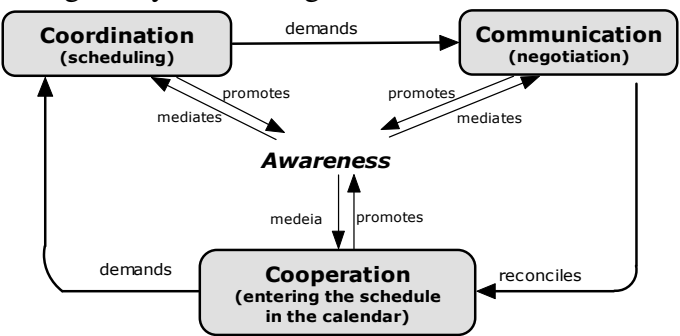

Figure 6. 3Cs in Family Calendar

Finally, the instance of the 3C model that characterizes Command and Control is presented (Figure 7). Here coordination is actually supervision, which oversees group production and commands changes. An example where command and control are collapsed is rowing, where the captain observes the performance of the rowers and shouts commands demanding more effort from those on one side.

These cycles show the iterative nature of collaboration. They also demonstrate how the 3Cs interact between themselves. These interactions are mediated by the awareness information available in the environment, which, in turn, influences and is influenced by each dimension of collaboration.

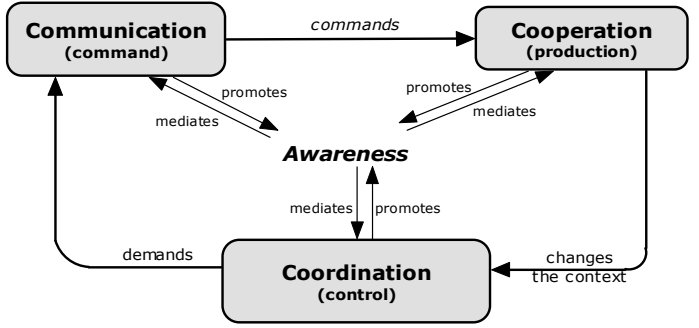

Figure 7. $3 \mathrm{Cs}$ in Command \& Control

Figure 8 presents how the examples are located inside the 3C triangle. The first $\mathrm{C}$ of the interrelationship is the vertex of the triangle closest to which the system is located, as for example, in the case of the adaptive workflow, coordination. The second $\mathrm{C}$ in the adaptive workflow cycle (Figure 3) is cooperation, which acts as an attractor in the direction of its vertex.

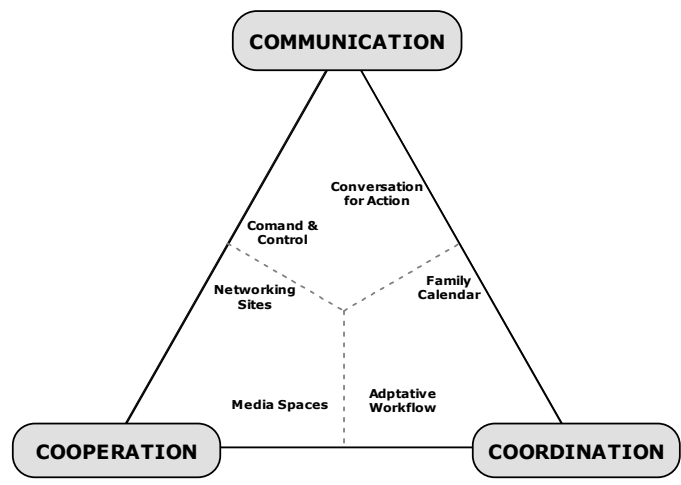

Figure 8. Collaboration Forms in the Triangle

Given that most of current collaborative systems show a greater bundling of functionality, no matter how directed to a certain $\mathrm{C}$ a groupware is, it probably also deals with the other 2Cs. For example, a message system such as the e-mail, albeit designed to establish conversation, can also be used to coordinate teamwork.

The inter-relationships of the 3Cs presented in this section illustrate how different application domains lead to different cycles of communication, coordination and cooperation acts, which the corresponding collaborative systems need somehow to support. In order to investigate how to provide this computational support, it is necessary to shift to a "microscopic" view of each collaborative service. In the next section, another form of relationships between the Cs is revealed. It is shown that the $3 \mathrm{Cs}$ interact within themselves.

\section{Inside each $\mathrm{C}$ there are the 3Cs: Intra- relationships}

In this section, the need to give support to the $3 \mathrm{Cs}$ 
inside each $\mathrm{C}$ will be investigated. For example, although the chat is a communication tool whose objective is to enable the exchange of messages among the members of a group, its use also includes acts of coordination and cooperation that are necessary to organize and record communication (Figure 9).

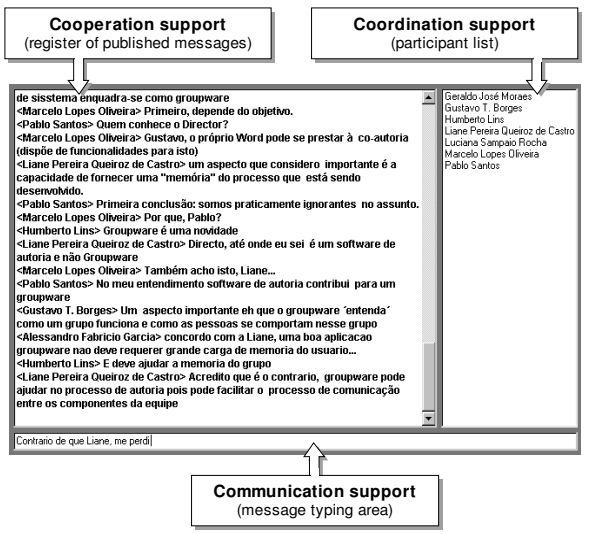

Figure 9. Chat areas for the $3 \mathrm{C}$ dimensions

In analyzing a typical chat tool, three areas are identified: a) an area containing a field to type the message, which enables the user to communicate with the other participants, constituting support to communication; b) an area containing a list of participants, indicating who is connected and available for conversation, constituting support to coordination; and c) an area presenting a log of messages sent, constituting support to cooperation.

The $3 \mathrm{C}$ collaboration model is used as a basis for the development of AulaNet, a web-based education and learning environment [6]. AulaNet services are divided into communication, coordination and cooperation services. In version 3.0 of this environment, services have been developed with the use of an architecture whose framework and components are based on the 3C model [8]. In this approach the model is also used to define the classification of components and services, bringing the implementation phase in line with other phases of the process that also make use of the $3 \mathrm{C}$ model: analysis, requirements and tests.

The course Information Technologies Applied to Education (ITAE), currently in its 17 th edition, which is given online through the AulaNet Environment, has in its initial phase a collaborative activity where the weekly topic is debated synchronously. The Debate service offers the adequate computational support for this activity. Originally, this service was based on a chat tool similar to that presented in Figure 9. In a typical chat tool, the only way to coordinate the debate is through the existing social protocol, where learners respect the moderator, him/herself also a learner, because of the (silent) presence of the course mediators. However, some difficulties that the standing social protocol could not fix appeared in the course's 10th edition when the dynamics of the debate changed.

The new debate dynamics is presented in Figure 10. The coordination is the responsibility of the mediators and comprises the opening, the closing of the debate, and the selection of the conversation technique. When mediators opt for a given conversational technique they let the conversation flow freely, in a circular form, or else allow the sending of a single message per learner (used in voting, for instance). They can also block the sending of messages by learners, including the moderator, at any moment. Communication is carried on by the learners, who edit and send messages. Cooperation, in the case of a chat, corresponds to the reading and presentation of messages (seminar, questions, votes, etc.). These activities: edition, submission, election, voting, etc., are not atomic and

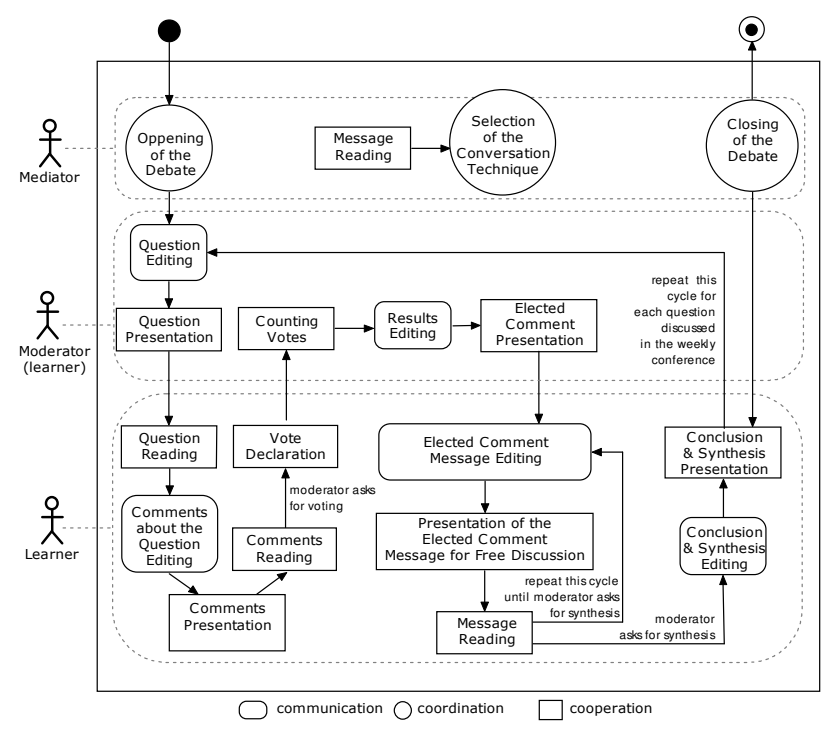

Figure 10. Debate Dynamics of the ITAE course 
could be subdivided, but once they are individual activities, they are out of the scope of this investigation.

Although the chat is a communication service, the newly added functionalities, such as mechanisms of conversational techniques, belong to coordination. This adaptability makes evident the strength of the model in the evolution of a collaborative system.

Figure 11 presents the evolution of the Debate service interfaces resulting from the new version of the Mediated Chat obtained through the process of development of Groupware Engineering based on the 3C collaboration model [7]. The development in successive versions is especially useful for collaborative systems once changes in the tool may generate unforeseen and undesirable modifications in the way a group works, calling for a revision of the solution implemented in a subsequent version. The development of each new version must be focused on a C. However, it is not enough to modify only one $\mathrm{C}$ per version, because when altering one of the functional dimensions of collaboration, it is necessary to be aware of the fact that the $\mathrm{Cs}$ are interdependent.

The mechanism of conversational techniques gave coordination computational support to the new dynamics. Through this mechanism, mediators define whether the conversation will be free, circular or single collaboration, or whether they will block the learners, keeping it accessible only to the mediators. Regarding communication, the maximum number of characters per message was increased and skipping a line using the ENTER key was enabled, creating the need for a specific button to trigger the sending of the message. In the registration area, which gives support to cooperation, all messages started to be preceded by their timestamp and the system messages by '***'. Finally, returning to coordination, in a typical chat only the list of the present participants is displayed, in the Mediated Chat the names of the mediators appear prominently.

\section{Discussion: Suitability of the $3 \mathrm{C}$ model to human perception}

In this section we discuss how members of a group alternate their attention between the $3 \mathrm{Cs}$ functionalities available in a groupware, thus demonstrating the suitability of this model to human perception.

It is observed in Figure 12 that, in spite of the alterations, these have been made in such way that the areas that give support to each $\mathrm{C}$ remain practically stable. There is no scattering of elements from a given $\mathrm{C}$ into the area of another $\mathrm{C}$, and, most importantly, these areas are visualized as clearly distinct blocks that make up the user interface. The groupings of the $3 \mathrm{C}$ mechanisms in well defined areas favor perception. Gestalt psychology [10] studies the capability of our senses to perceive forms, especially regarding the visualization of complete figures, preferably closed, as opposed to a set of lines and curves.

Another principle of Gestalt psychology used in this investigation is Figure and Ground [17]. In certain images, a person perceives that one part emerges (becomes figure) and, while our attention is concentrated on it, the remaining part of the image disappears (constituting the ground). When part of this ground emerges becoming figure, the previous figure becomes part of the ground. McLuhan [15] extends this principle to the entire structure of perception and conscience: in all situations there is an area of attention (figure) and another larger area of inattention (ground), and these areas interact continuously with each other through a common border that defines both simultaneously- "Each new figure displaces the previous one back to the ground". In the case of the Debate service, between the finishing of a phase and the beginning of another one (such as, for example, finishing typing a message and consulting the log of published messages), there is a displacement of attention: what was figure (communication) becomes ground, and part of the previous ground becomes the current figure (cooperation). McLuhan states that "in the natural order of things, the ground comes first, and the figure emerges later". In other words, without the ground there is no figure, a realization that reinforces the conviction that the $3 \mathrm{Cs}$ are needed for collaboration.

\section{Conclusion}

In order to support a variety of roles, tasks, and working modes, most of current collaborative systems encompass a large range of functionalities. In this context, the investigation of how these functionalities interact is richer than looking at each one of them in isolation. Starting from the static 3C model, used to functionally classify collaborative systems, this paper

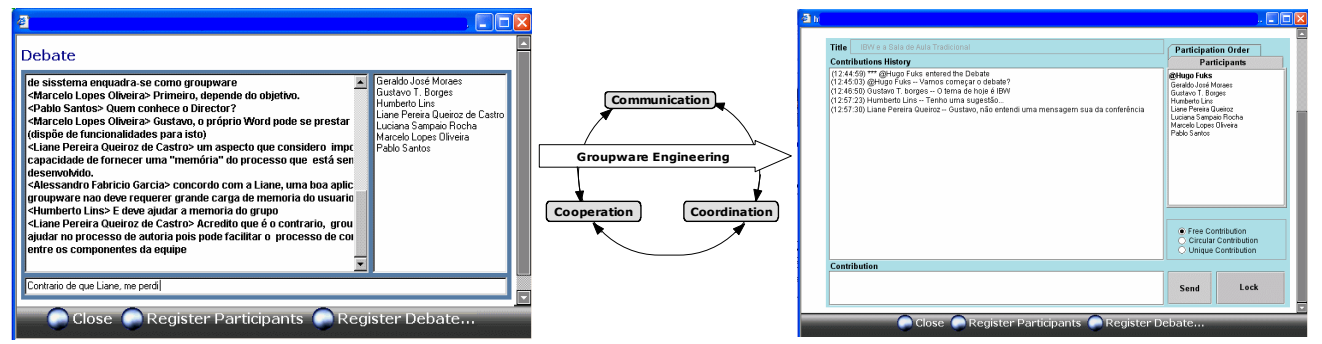

Figure 11. Previous and current user interfaces for the Debate service in AulaNet 

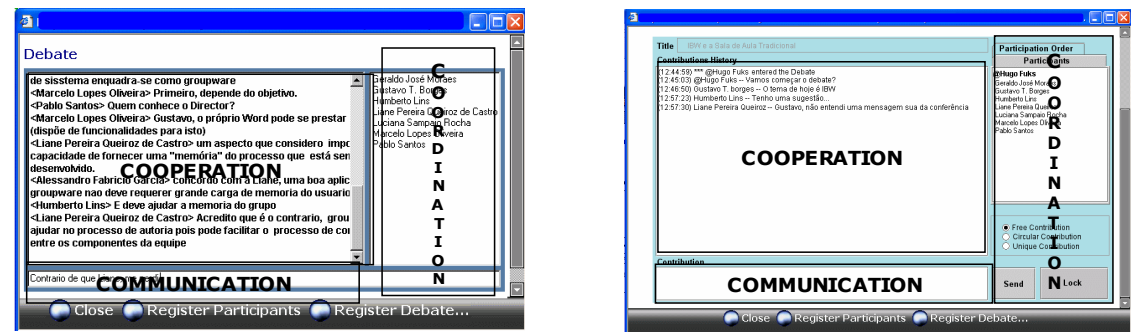

Figure 12. Distinct blocks for each $\mathrm{C}$ in the Debate service user interface

discusses the inter- and intra-relationships between communication, coordination, and cooperation. Interrelationships provide a mapping for a variety of collaboration forms, while intra-relationships reveal the recurrent nature of collaboration. It was discussed the suitability of collaborative systems engineered by the $3 \mathrm{C}$ model to human perception in the light of Gestalt psychology principles. Finally, all instances of the (inter-) 3C model here described are passive of implementation using component-based development techniques based on (intra-) components [8].

\section{Acknowledgements}

The AulaNet project is partially sponsored by Fund. Pe. Leonel Franca and by the MCT through the ESSMA project grant $\mathrm{n}^{\circ} 552068 / 2002-0$. It is also sponsored by CNPq individual grants: C. Lucena $n^{\circ}$ 300091/2003-6, H. Fuks no 301917/2005-1, M. Gerosa no 383719/06-2. C. Lucena and H. Fuks also receive grants from the FAPERJ project "Cientistas do Nosso Estado". D. Filippo receives a grant CCPG/VRAc from PUC-Rio.

\section{References}

[1] S. Bandinelli, E. D. Nitto and A. Fuggetta, "Supporting cooperation in the SPADE-1 Environment", IEEE Trans. on Software Engineering, 1996, 22(12), 841-865.

[2] U. M. Borghoff and J. H. Schlichter, ComputerSupported Cooperative Work: Introduction to Distributed Applications. Springer, USA, 2000.

[3] I. Bretain, L. Fredin, W. Frost, L. R. Hedman, P. Kroon, S. McGlashan, E. L. Sallnas and M. Virtanen, "Leave the Office, Bring Your Colleagues: Design Solutions for Mobile Teamworkers", Proc. SIGCHI conference on Human Factors in Computing Systems (CHI), ACM Press, New York, NY, 1997, 335-336.

[4] K. Elliot and S. Carpendale, "Awareness and Coordination: A Calendar for Families". Technical Report 2005-791-22, Dept. of Computer Science, Univ. of Calgary, Canada, T2N 1N4, 2005.

[5] C. A. Ellis, S. J. Gibbs, and G. L. Rein, "Groupware Some Issues and Experiences", Communications of the ACM, 1991, 34(1),. 38-58.

[6] H. Fuks, M. A. Gerosa and C. J. P. Lucena, "The Development and Application of Distance Learning on the Internet. Open Learning", The Journal of Open and Distance Learning, 2002, 17(1), 23-38.

[7] H. Fuks, A. B. Raposo, M. A. Gerosa and C. J. P. Lucena, "Applying the $3 \mathrm{C}$ Model to Groupware
Development", International Journal of Cooperative Information Systems, 2005, 14(2-3), 299-328.

[8] H. Fuks, M. Pimentel and C. J. P. Lucena, "R-U-Typing2-Me? Evolving a chat tool to increase understanding in learning", International Journal of Computer-Supported Collaborative Learning, 2006, 1(1), 117-142.

[9] W. Gaver, T. Moran, A. MacLean, L. Lövstrand, P. Dourish, K. Carter, and W. Buxton, "Realizing a video environment: EuroPARC's RAVE system”, Proc. SIGCHI conference on Human Factors in Computing Systems, (CHI) ACM Press, New York, 1992, 27-35.

[10] W. Köhler, Gestalt Psychology: An Introduction to New Concepts in Modern Psychology, Liveright Publishing Company, NY, 1947 (reprint 1992).

[11] Y. Laurillau and L. Nigay, "Clover architecture for groupware", Proc. ACM Conference on ComputerSupported Cooperative Work (CSCW), ACM Press, New York, NY, 2002, 236-245.

[12] W. E. Mackay, "Media Spaces: environments for Informal Multimedia Interaction", In Computer Supported Co-operative Work (Trends in Software: 7), Beaudouin-Lafon, M. (ed). John Wiley \& Sons, England, 1999, 55-82.

[13] M. Magnusson and L. Svensson, "Studying how students study: Work-orientation and collaboration in Distance Education", Proc. IRIS 23, Univ. of Trollhättan Uddevalla, Sweden, 2000.

[14] T. W. Malone and K. Crowston, "The interdisciplinary study of coordination", ACM Computing Surveys, 1994, 26(1), 87-119.

[15] M. McLuhan and E. McLuhan, Laws of Media: The New Science, University of Toronto Press, Toronto, 1988.

[16] D. C. Neale, J. M. Carroll and M. B. Rosson, "Evaluating Computer-Supported Cooperative Work: Models and Frameworks", Proc. ACM Conference on Computer-Supported Cooperative Work (CSCW), ACM Press, New York, NY, 2004, 112-121.

[17] E. Rubin, "Figure and ground", In Readings in perception, D. C. Beardslee and M. Wertheimer (eds). Van Nostrand: Princeton, NJ, 1958.

[18] B. Weber, W. Wild and R. Breu, "CBRFlow: Enabling Adaptive Workflow Management through Conversational Case-Based Reasoning", LNCS 3155, Springer, Berlin/Heidelberg, 2004, 434-438.

[19] P. E. Weiseth, B. E. Munkvold, B. Tvedte. and S. Larsen, "The Wheel of Collaboration Tools: A Typology for Analysis within a Holistic Framework", Proc. ACM Conference on Computer-Supported Cooperative Work $(C S C W)$. ACM Press, New York, 2006, 239-248. 